\title{
The design of ceramic roller kiln pulse combustion controller
}

\author{
Wenliang Xie ${ }^{a}$, Jing Chen ${ }^{b}$, Youxin Yuan ${ }^{c}$, Baoping Mao ${ }^{d}$ \\ School of Automation, Wuhan University of Technology, Wuhan China, 430000 \\ a1512525691@qq.com, bjingchen680@163.com, cyyx2000@263.net
}

Keywords: ceramic roller kiln, pulse combustion, controller

\begin{abstract}
The ceramic industry is one of Chinese symbolic industries. From ancient times, the ceramic industry has been the project focused and researched by the government. Because of the small temperature difference, energy-saving, high-level automation and so on, ceramic roller kiln has been widely used in many domestic ceramics production industries. For these ceramic roller kiln's characters, designing an effective combustion way is necessary. Under the Ministry of Science and Technology support project "the pure-oxygen combustion technology and equipment of ceramic kilns (2012BAA08B04)" and our research group, the overall design, the hardware design and the software design are put forward for the ceramic roller kiln pulse combustion controller.
\end{abstract}

\section{Introduction}

The ceramic has been the gem of our country from the ancient time. The archaeologist found that Chinese has made ceramic from the Neolithic Age. Because the ceramic is the main part of export productions, the government shows great expectations for it.[1]

Our group has done many researches about the combustion technology and equipment of ceramic kiln in recent years, and has advanced level in the country. This project got strongly supports from the Science and Technology project of Hubei province, the national Ministry of science and technology project, the Ministry of Science and Technology support project and ceramics production enterprises. Our group not only published a number paper and applied for a great deal of inventions and novel utility patents, but also fabricated a great deal of control systems for enterprises.

But so far, our group hasn't had an intensive study about combustion mode. Literature [2]-[5] focuses on the introduction of novel pulse combustion mode and the structure description of hybrid combustion control system. About the combustion project and controller in industry production, our group hasn't had an effective proposal yet. To solve this problem, a scheme about ceramic roller kiln pulse combustion controller is put forward in this paper.

\section{Design scheme of pulse combustion controller}

Producing the PWM wave to control the impulse burner is the key to make pulse combustion controller. Because of the big sectional area and long firing zone of the ceramic roller kiln, premixed high-speed pulse burner is used in the system. This burner required signal current regularly is several hundred on work. Therefore, the signal produced by ordinary SCM is unable to be used directly. A PWM drive circuit is needed in this system to transfer the small current signal to high current signal.

Based on our group research experiences, our group found that driver circuit ordinarily is made up of integrated IC or is bridge driver circuit. The integrated IC such as LM298 series is generally used in the control of small power DC motor. But the power and driver current of these chips are unable to achieve the requirement. The pulse burner controller just control the on and off of the current, therefore the half bridge circuit is used in the driver of this system. The whole driver circuit is consists of MOSFET circuit, protect circuit, crowbar circuit and so on. In order to simplify the design and reduce the cost, the half bridge driver chip IR2110 with isolation function produced by 
IR Company is used in this system.

The principal tasks of the main controller of this system are accomplishing the flow collection of gas and oxygen, receiving the duty cycle signals from upper monitor, controlling the on and off of the gas and oxygen and producing the PWM signals pulse burner need. According to the requirement and cost, our group use STC12C4052AD as main controller.

For the sake of the security of the system, electromagnetic valves have been set on the gas and oxygen pipelines. When the burner is not on work, the electromagnetic valves would keep the gas and oxygen pipelines off. While the burner is out-of-order, the pulse combustion controller would give sound and light alarm to warn user that this combustion module goes wrong.

\section{The hardware design of pulse combustion controller}

The detection of the gas and oxygen flow is not necessary in the pulse combustion system. But in order to make the pressure of the pulse burner from the gas pipeline detected convenient, AWM2000 series gas flow indicator is used in this system in order to detect the gas and oxygen flow.

The single CPU control scheme is used in this system, in order to save cost and reduce the plate area of the controller. The PWM signals from controller are unable to drive the pulse burners directly. The proper driver is used on the pulse burner to improve the driving capacity of PWM signals. The pulse combustion controller receives duty cycle information of the burners on control through the RS485 bus. The pulse combustion controller not only gleans the flow of gas and oxygen to control the pipelines, but also produce PWM signals to control the pulse burner based on the duty cycle information from bus.

The total design scheme of the pulse combustion system hardware controller is shown in Fig. 1

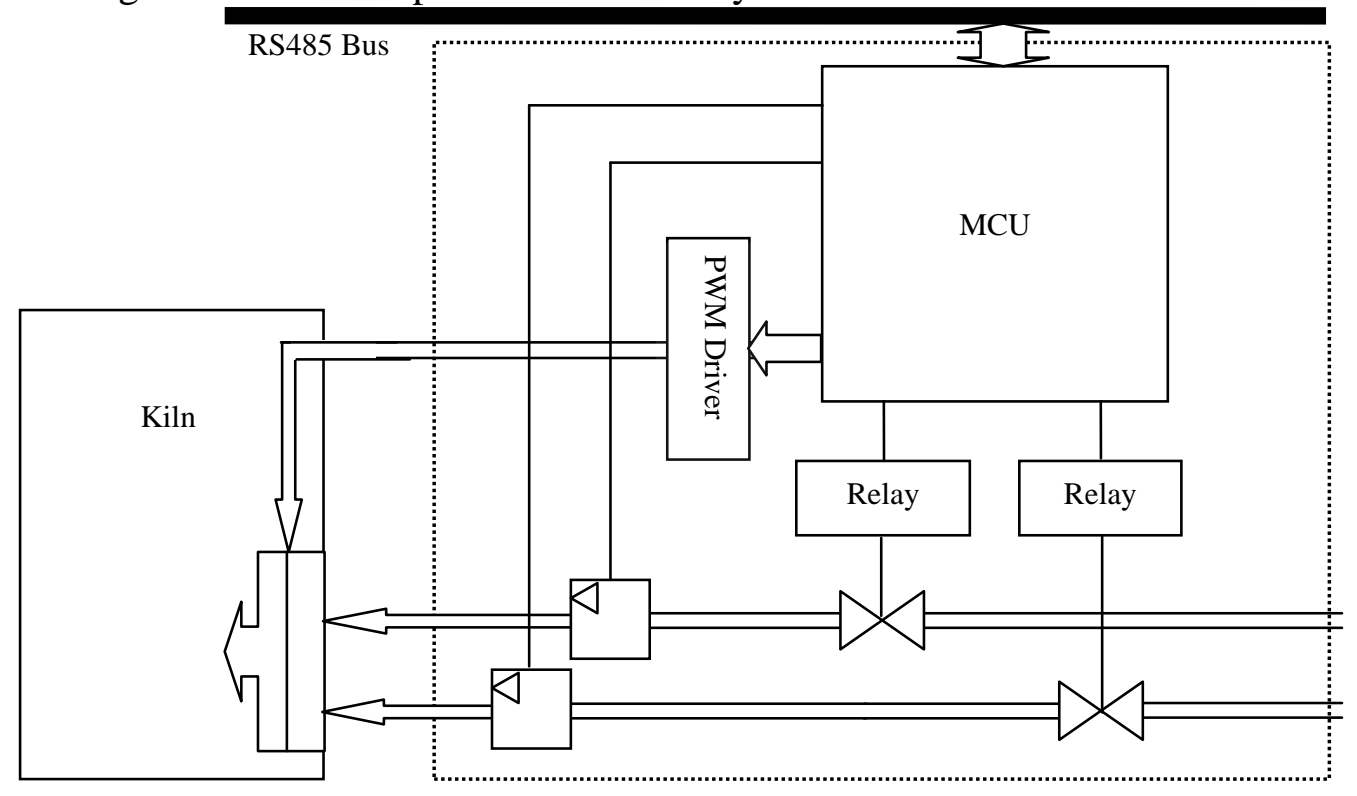

Fig.1, Pulse combustion controller design scheme block

\section{The software design of pulse combustion controller}

The system main controller mend temperature curve based on PID algorithm. The main controller used RTU method to communicate with the temperature and gas detection module based on Modbus protocol. In order to enhance the communication performance of this system, the data switching between the main controller and host computer is through the Ethernet. In this way, the monitor layer could be distributed in the factor, to achieve that multiple-hosts control multiple ceramic roller kiln pulse combustion system. In this system, the Ethernet modules integrate MAC and Ethernet physical layer devices.

The main controller of this system is consists of SD card module, Ethernet interface, and an 
external SD card module to extend storage. In each combustion period, the SD card records the related combustion information and control orders. After several periods (in this system it's 10), an expert system database would be set in the SD card. When the lost communication happened between host computer and main controller, the main controller could search the similar control data from SD card to maintain the system stable.

The pulse combustion controller is in the control of bus signals. The main control program flow chart of pulse combustion module is shown in Fig.2.

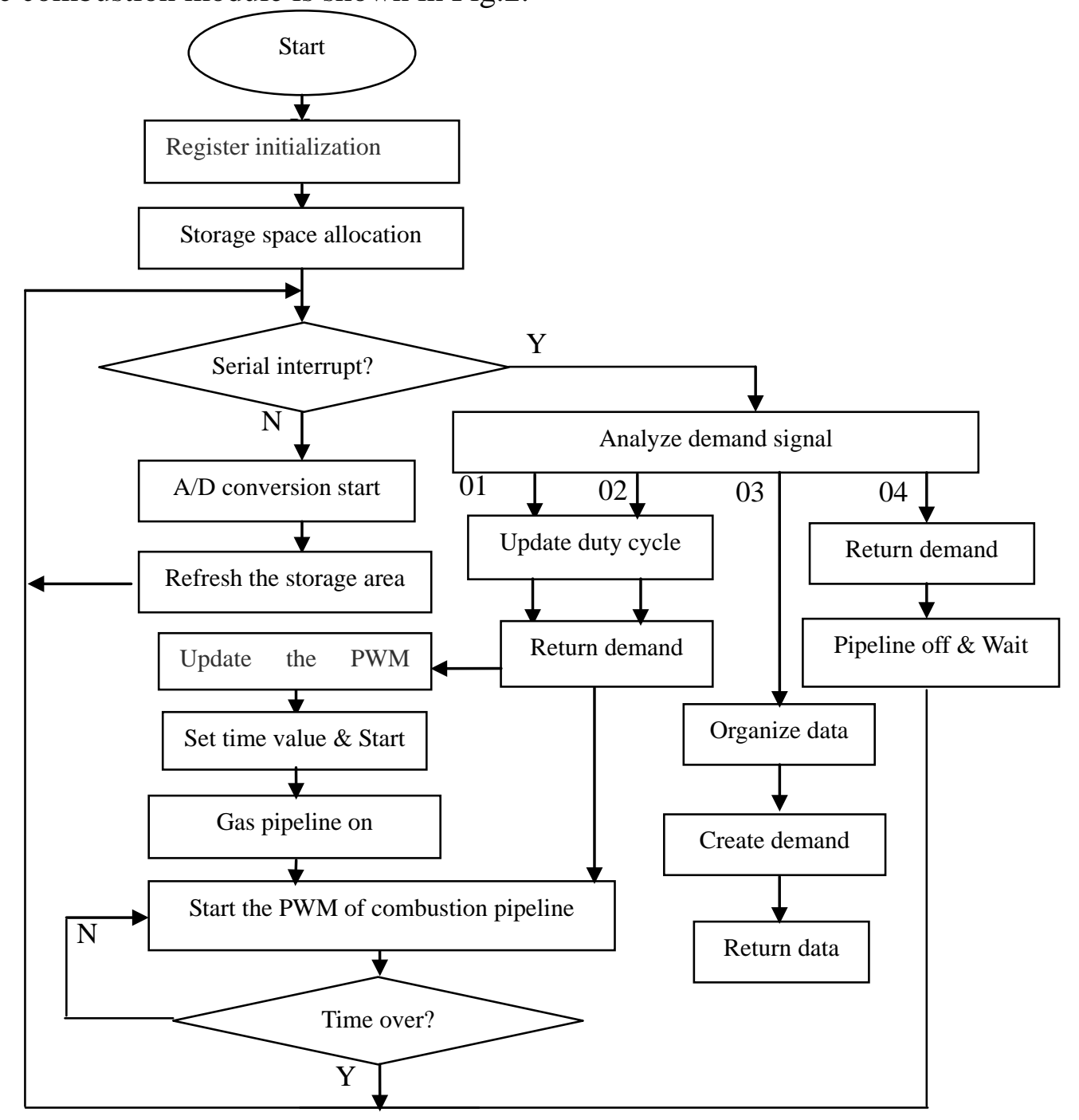

Fig.2, Main control program of pulse combustion module block diagram

\section{Conclusions}

A design scheme of ceramic roller kiln pulse combustion controller is put forward in this paper based on our group's research. And the hardware and software design scheme of the controller are introduced in this paper. The design scheme is proved to have favorable effect based on actual research.

\section{Acknowledgement}

The paper is supported by the Ministry of Science and Technology support project "The pure-oxygen combustion technology and equipment of ceramic kilns (2012BAA08B04)", and "Graduate innovation fund of Wuhan University of Technology (125211006)”. 


\section{References}

[1] Zhili Peng,Jun Bo, The development history Chinese ceramic kiln[C]. History of Mechanical Technology-- The first China Japan International Conference on history of Mechanical Technology Proceedings, 1998, I: 176-182.

[2] Jing Chen, Bihui Huang, Youxin Yuan, Chun Xiao, Kui Xiang. Pulse Combustion Control Technology for Oxygen Atmosphere[C]. Proceedings of 2009 International IEEE Workshop on Intelligent Systems and Applications, pl851. 1853(EI accession NO.:20094112366852).

[3] Jing Chen, Jianxun Lang, Yuan Youxin, Chun Xiao, Bihui Huang, The Design of Ceramic Roller Kiln Pulse Combustion System Based on Fuzzy Control[C]. Proceedings of 2009 China Intelligent D Automation Conference, 2009,9,27-29,I: 371-375.

[4] Baoping Mao, Youxin Yuan, Chun Xiao, Zhuo Chen, The Pulse Combustion Control Device of Pure-oxygen Ceramic Roller Kiln[P].China: 201320219751.3. 2013-04-26.

[5] Jing Chen, Jia Li, The Pure-oxygen combustion ceramic roller kiln control system[J]. Journal of Wuhan University of Technology, 2010,32(11): 86-89 